\title{
eRHIC - an Electron-Ion Collider at BNL
}

\section{Christoph Montag*’, Brookhaven National Laboratory, Upton, NY 11973, USA}

E-mail: montagebnl.gov

\begin{abstract}
The electron-ion collider eRHIC aims at a luminosity around $10^{34} \mathrm{~cm}^{-2} \mathrm{sec}^{-1}$. The design is based on the existing Relativistic Heavy Ion Collider (RHIC) facility, which has successfully accelerated, stored and collided polarized proton beams of up to $255 \mathrm{GeV}$ as well as ions up to $100 \mathrm{GeV} / \mathrm{n}$ beam energy. A 5 to $18 \mathrm{GeV}$ electron storage ring will be added in the RHIC tunnel, thus providing electron-polarized proton or electron-ion collisions in up to two interaction regions. A spin-transparent rapid-cycling synchrotron (RCS) will serve as full energy polarized electron injector, thus enabling arbitrary spin patterns in the electron storage ring.
\end{abstract}

23rd International Spin Physics Symposium - SPIN2018 -

10-14 September, 2018

Ferrara, Italy

* Speaker.

$\dagger$ This document has been authorized by employees of Brookhaven Science Associates, LLC under Contract No. DE-SC0012704 with the U.S. Department of Energy. The United States Government retains a non-exclusive, paid-up, irrevocable, world-wide license to publish or reproduce the published form of this document, or allow others to do so, for United States Government purposes. 


\section{Introduction}

The Relativistic Heavy Ion Collider (RHIC), depicted in Figure 1, consists of two superconducting storage rings ("BLUE" and "YELLOW") that intersect at six equidistantly spaced interaction regions arond their circumference. These interaction regions (IRs) are named according to their location resembling the face of a clock, with "12 o'clock" (or IR12) being the northernmost IR. Two of these IRs are currently equipped with detectors - STAR in IR6, and PHENIX in IR8. RHIC is capable of accelerating and colliding virtually any ion species, from protons to fully stripped uranium. The maximum proton beam energy is $255 \mathrm{GeV}$, which in terms of beam rigidity $B \rho$ is equivalent to $100 \mathrm{GeV} / \mathrm{n}$ gold. A unique feature of RHIC is the fact that proton beams are spin polarized, with polarization levels exceeding 60 percent at $255 \mathrm{GeV}$, which makes RHIC the only polarized proton collider in the world.

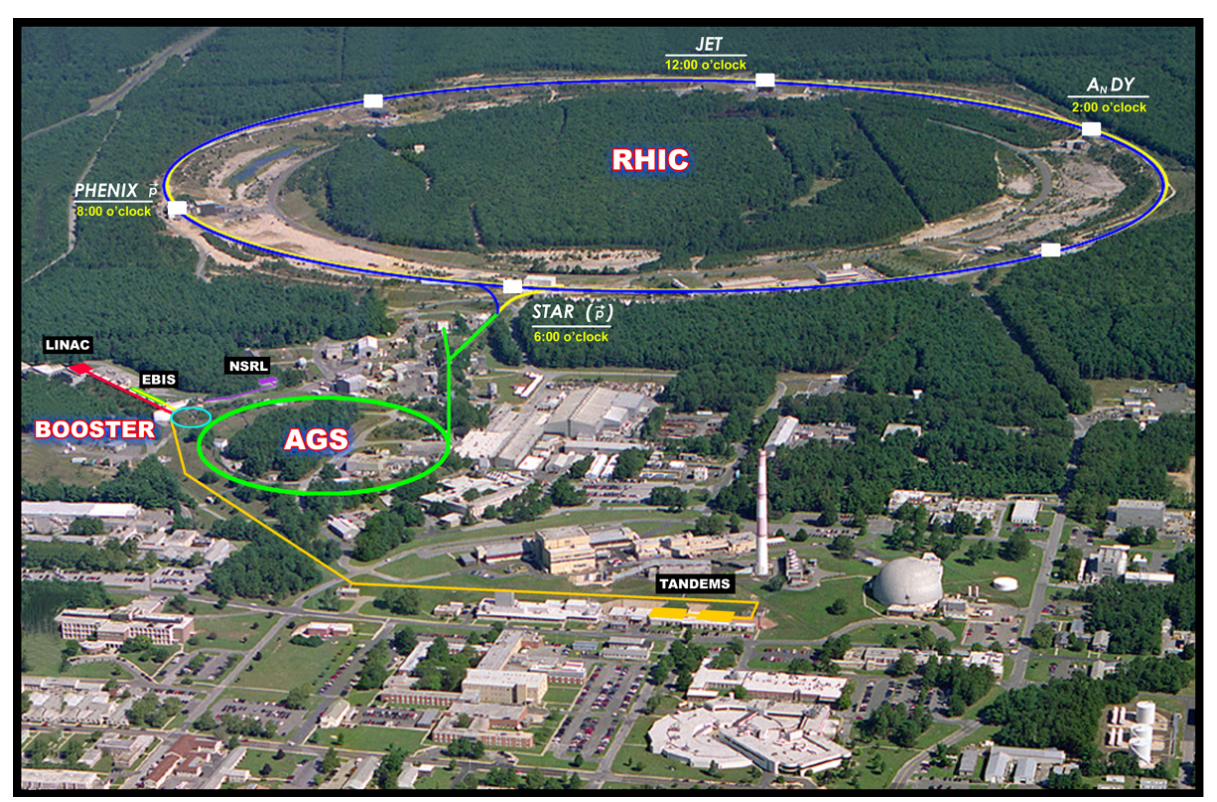

Figure 1: Overview of the RHIC facility with its injector complex

In response to the recommendation by the US nuclear physics community for a polarized electron-ion collider formulated in the White Paper [1], Brookhaven National Laboratory (BNL) is proposing eRHIC. eRHIC is designed to meet all the requirements formulated in the White Paper:

- High luminosity of $10^{33}$ to $10^{34} \mathrm{~cm}^{-2} \mathrm{sec}^{-1}$;

- A large range of center-of-mass energies from 29 to $140 \mathrm{GeV}$;

- Polarized electron and light ion (protons, ${ }^{3} \mathrm{He}, \ldots$ ) beams with flexible spin patterns;

- Favorable conditions for detector acceptance, such as $p_{t}$ acceptance down to $200 \mathrm{MeV} / \mathrm{c}$;

- A large range of hadron species, from protons to uranium. 
The design concept of the machine is based on the existing RHIC complex, namely the "YELLOW" storage ring, the entire ion injector complex, and infrastructure including the $3.8 \mathrm{~km}$ circumference tunnel, all of which only needs few modifications and upgrades to be suitable for eRHIC. Except for the number of bunches, the total beam current, and the vertical beam emittance, present RHIC beam parameters are close to what is required to reach a peak luminosity of $10^{34} \mathrm{~cm}^{-2} \mathrm{sec}^{-1}$. eRHIC would be realized by adding an electron storage ring with an energy range from 5 to $18 \mathrm{GeV}$ in the RHIC tunnel, and colliding the polarized electron beam with the ion beam stored in the "YELLOW" ring. The total synchrotron radiation has been limited to $10 \mathrm{MW}$. This is purely a design choice, not a technical limitation, as the linear synchrotron radiation load of $4 \mathrm{~kW} / \mathrm{m}$ in the arcs is more than a factor 2 below what has been achieved at KEKB [2]. The design of the entire collider facility is optimized under the assumption that each beam will reach the parameters - in particular the beam-beam tune shift - as demonstrated in collisions between equal species. This concept was successfully applied in the design of HERA [3]. To allow storage of electron beams with variable spin patterns, a full-energy polarized injector is required. This is realized by a spintransparent rapid-cycling synchrotron (RCS) [4] in the existing RHIC tunnel which accelerates polarized electron bunches with intensities up to $10 \mathrm{nC}$ from $400 \mathrm{MeV}$ to a maximum energy of $18 \mathrm{GeV}$. Figure 2 shows a rendering of the eRHIC facility.

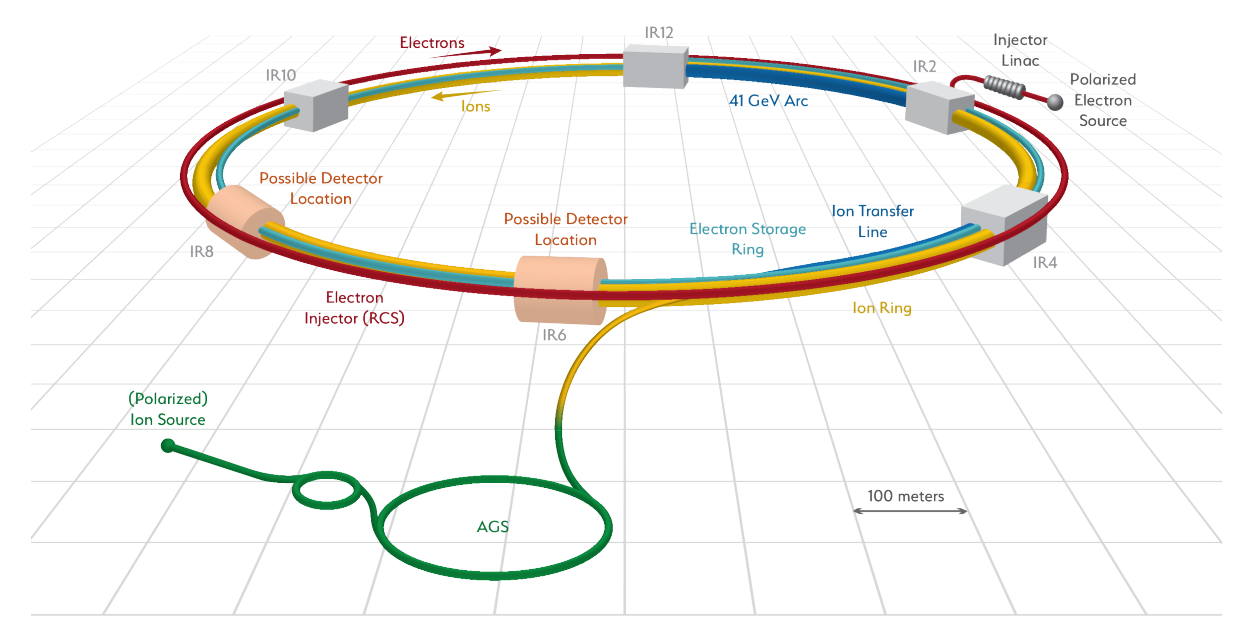

Figure 2: eRHIC facility layout in the existing RHIC tunnel

\section{Maximum luminosity parameters}

eRHIC reaches its maximum luminosity of $1.05 \times 10^{34} \mathrm{~cm}^{-2} \mathrm{sec}^{-1}$ at a center-of-mass energy of $\sqrt{s}=105 \mathrm{GeV}$, by colliding $10 \mathrm{GeV}$ electrons with $275 \mathrm{GeV}$ protons. This high luminosity is achieved by a combination of high beam currents in a large number of bunches, large beam-beam tune shifts, small vertical emittances, and short hadron bunches. The limits on the electron beam parameters are chosen according to the achievements at the B-factories CESR-B [5], KEKB [6], and PEP-II [7, 8], see Table 1. For instance, the number of bunches in eRHIC is similar to KEKB 
Table 1: Comparison of the eRHIC electron storage ring with the B-factories.

\begin{tabular}{lcccc}
\hline \hline & CESR-B & PEP-II & KEKB & \\
& & LER/HER & LER/HER & \\
\hline circumference $[\mathrm{m}]$ & 768 & 2200 & 3000 & 3834 \\
no. of bunches & 36 & 1588 & 1584 & 1320 \\
beam current $[\mathrm{A}]$ & 0.365 & $2.45 / 1.55$ & $1.3 / 1.6$ & 2.5 \\
bunch intensity $\left[10^{10}\right]$ & 16.2 & $7.0 / 4.4$ & $5.0 / 6.2$ & 15 \\
beam-beam parameter & 0.062 & $0.064 / 0.055$ & $0.12 / 0.1$ & 0.1 \\
transv. damping decrement $\left[10^{-4}\right]$ & 1.1 & $1.8 / 2$ & 2.5 & 1.25 \\
\hline \hline
\end{tabular}

Table 2: Proton beam emittances and intensities achieved during routine RHIC operations.

\begin{tabular}{lc}
\hline \hline bunch intensity $\left[10^{10}\right]$ & 26 \\
RMS normalized emittance, $\mathrm{h} / \mathrm{v}[\mu \mathrm{m}]$ & $2.5 / 2.5$ \\
longitudinal bunch area $[\mathrm{eV} \cdot \mathrm{sec}]$ & 1.6 \\
\hline \hline
\end{tabular}

in a similarly sized ring, while the total beam current is essentially the same as what has been achieved in the PEP-II low energy ring. The bunch intensity in eRHIC is comparable to CESR-B, while the maximum beam-beam parameter is close to the value reached at KEKB.

The horizontal emittance and intensity of the proton beam in eRHIC are similar to routine operations parameters at RHIC (see Table 2). However, the required vertical emittance is significantly smaller. Together with the short bunch length this results in an IBS growth time of roughly $2 \mathrm{~h}$, and requires strong electron cooling to first achieve and then preserve the small emittance over the entire duration of a physics store. Table 3 lists the eRHIC beam parameters for maximum luminosity, while Figure 3 shows the peak luminosity vs. center-of-mass energy.

Multiple approaches for strong hadron cooling are currently under consideration. Due to the high beam energy, conventional electron cooling with a DC beam is not applicable. Instead, an $\mathrm{RF}$ accelerator is required to accelerate electron beams to energies between 20 and $150 \mathrm{MeV}$, thus matching the Lorentz- $\gamma$ of the hadron beams. The most promising concept is some variant of coherent electron cooling $[9,10]$. In this scheme, a bunched electron beam is merged with the stored hadron beam over a length of several meters. Longitudinal fluctuations of the hadron beam density get imprinted on the electron beam, which thus serves as a pick-up. These fluctuations are subsequently amplified in an FEL or a plasma cascade, and the electron beam is then merged with the hadron beam a second time here it serves as a kicker to correct the density fluctuations of the hadron beam. The whole process therefore resembles a stochastic cooling system with a very large bandwidth in the $\mathrm{THz}$ range, resulting in short cooling time constants. 
Table 3: Beam parameters for maximum luminosity.

\begin{tabular}{lcc}
\hline \hline parameter & proton & electron \\
\hline center-of-mass energy [GeV] & \multicolumn{2}{c}{105} \\
energy [GeV] & 275 & 10 \\
number of bunches & \multicolumn{2}{c}{1320} \\
particles per bunch [10 ${ }^{10}$ ] & 6.0 & 15.1 \\
beam current [A] & 1.0 & 2.5 \\
horizontal emittance [nm] & 9.2 & 20.0 \\
vertical emittance [nm] & 1.3 & 1.0 \\
hor. $\beta$-function at IP [cm] & 90 & 42 \\
vert. $\beta$-function at IP [cm] & 4.0 & 5.0 \\
hor./vert. fractional tunes & $0.3 / 0.31$ & $0.08 / 0.06$ \\
RMS hor. divergence at IP [mrad] & 0.101 & 0.219 \\
RMS vert. divergence at IP [mrad] & 0.179 & 0.143 \\
hor. beam-beam parameter & 0.013 & 0.064 \\
vert. beam-beam parameter & 0.007 & 0.10 \\
IBS growth time long./hor. [h] & $2.19 / 2.06$ & N/A \\
synchrotron radiation power [MW] & N/A & 9.18 \\
RMS bunch length [cm] & 5 & 1.9 \\
hourglass and crab reduction factor & \multicolumn{2}{c}{0.87} \\
luminosity [10 ${ }^{34} \mathrm{~cm}^{-2}$ sec ${ }^{-1}$ ] & \multicolumn{2}{c}{1.05} \\
\hline \hline
\end{tabular}

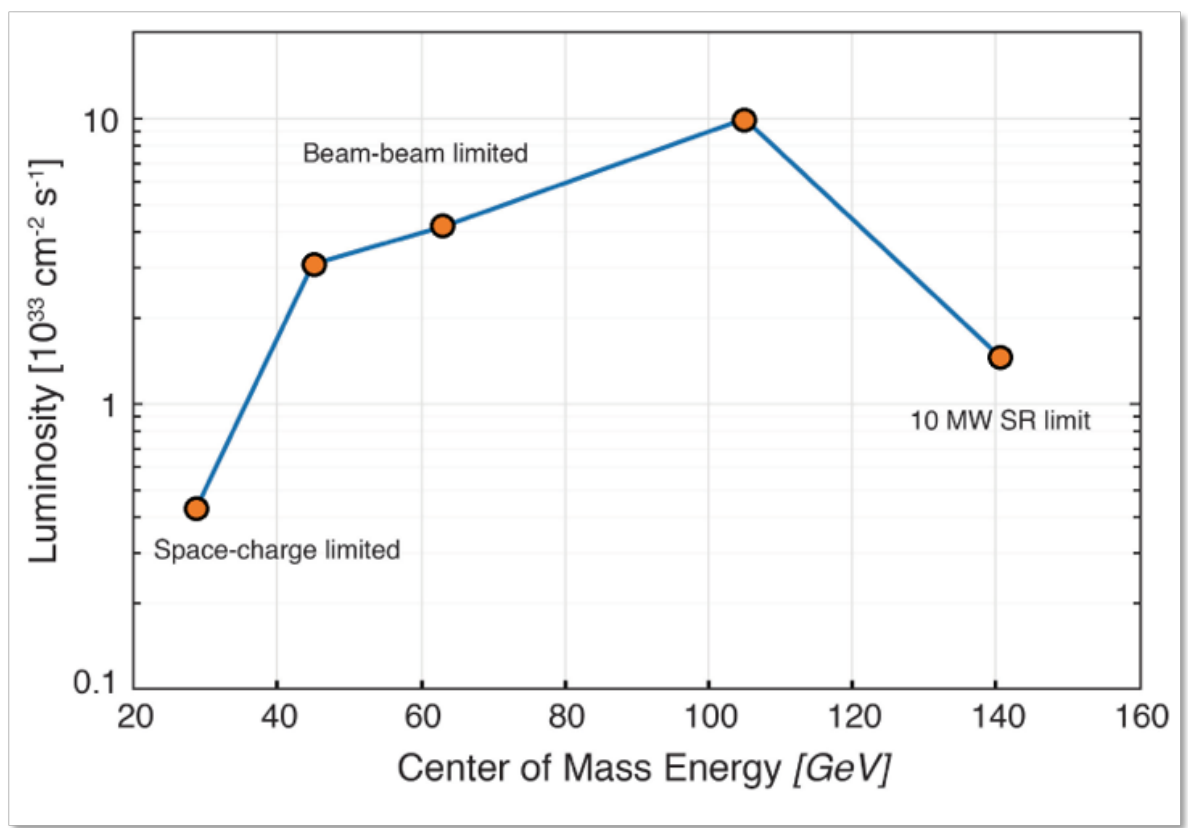

Figure 3: Luminosity vs. center-of-mass energy 
Table 4: Beam parameters without strong hadron cooling.

\begin{tabular}{lcc}
\hline \hline parameter & proton & electron \\
\hline center-of-mass energy [GeV] & \multicolumn{2}{c}{105} \\
energy [GeV] & 275 & \multicolumn{2}{c}{10} \\
number of bunches & \multicolumn{2}{c}{660} \\
particles per bunch [10 ${ }^{10}$ ] & 10.5 & 30 \\
beam current [A] & 0.87 & 2.5 \\
horizontal emittance [nm] & 13.9 & 20.0 \\
vertical emittance [nm] & 8.5 & 4.9 \\
hor. $\beta$-function at IP [cm] & 90 & 63 \\
vert. $\beta$-function at IP [cm] & 5.9 & 10.4 \\
hor./vert. fractional tunes & $0.3 / 0.31$ & $0.08 / 0.06$ \\
RMS hor. divergence at IP [mrad] & 0.124 & 0.179 \\
RMS vert. divergence at IP [mrad] & 0.380 & 0.216 \\
hor. beam-beam parameter & 0.015 & 0.1 \\
vert. beam-beam parameter & 0.005 & 0.083 \\
IBS growth time long./hor. [h] & $10.1 / 9.2$ & N/A \\
synchrotron radiation power [MW] & N/A & 9.1 \\
RMS bunch length [cm] & 7 & 1.9 \\
luminosity [10 3 cm $^{-2}$ sec ${ }^{-1}$ ] & \multicolumn{2}{c}{4.4} \\
\hline \hline
\end{tabular}

\section{Mitigation of strong hadron cooling risk}

Strong hadron cooling at high energies, as it is required to reach the full $10^{34} \mathrm{~cm}^{-2} \mathrm{sec}^{-1}$ luminosity potential of eRHIC, has never been realized and therefore constitutes a high risk in the design concept. To mitigate this risk, an alternative parameter set has been worked out with a minimum IBS growth time of about 9 to $10 \mathrm{~h}$, as shown in Table 4 . This IBS growth time is similar to present RHIC, which has typical store durations of 10 to $12 \mathrm{~h}$ - sufficiently long compared to the turnaround time of 1 to 2 hours between stores.

This parameter set is based on hadron beam emittances that are similar to present RHIC. The design bunch length of $7 \mathrm{~cm}$ can be achieved by splitting regular high intensity RHIC bunches with the current longitudinal emittance of $1.6 \mathrm{eV} \cdot \mathrm{sec}$ to achieve the desired intensity and longitudinal emittance. As a result, eRHIC reaches a peak luminosity of $4.4 \times 10^{33} \mathrm{~cm}^{-2} \mathrm{sec}^{-1}$ at a center-ofmass energy of $105 \mathrm{GeV}$, as listed in Table 4.

\section{Electron storage ring}

The eRHIC electron storage ring is based on conventional FODO cells with a cell length of approximately $16 \mathrm{~m}$. To facilitate the large beam-beam tune shift parameter of $\xi=0.1$ at all energies, fast synchrotron radiation damping is required. While the damping decrement at $10 \mathrm{GeV}$ and beyond is sufficient, lower energies require special measures. This is accomplished by realizing 
the bending magnets in the arcs as so-called super-bends. These super-bends consist of two $2.6 \mathrm{~m}$ long dipoles with a short, $0.7 \mathrm{~m}$ long dipole in-between. At $10 \mathrm{GeV}$ and above, all magnets are powered uniformly, thus maximizing the bending radius and therefore minimizing the synchrotron radiation losses. Below $10 \mathrm{GeV}$, the polarity of the center dipole is reversed, resulting in a small local bending radius and therefore the generation of additional synchrotron radiation that leads to fast radiation damping.

To reach the design luminosity at all energies, the horizontal electron beam emittance needs to be nearly constant at 20 to $22 \mathrm{~nm}$ over the entire range. This is realized by varying the betatron phase advance per FODO cell. At $18 \mathrm{GeV}$, the phase advance is set to 90 degrees per cell, while at 10 and $5 \mathrm{GeV}$ it is set to 60 degrees. Together with the super-bends at $5 \mathrm{GeV}$, this results in the desired emittances.

The maximum synchrotron radiation power in the ring has been limited to $10 \mathrm{MW}$. This is a design choice, not a technical limit. 11 superconducting 2-cell RF frequencies at $563 \mathrm{MHz}$ will be installed to replenish those synchrotron radiation losses and provide the necessary longitudinal focusing.

\section{Hadron ring modifications}

A small number of modifications have to be carried out in the hadron storage ring to make it suitable for eRHIC. The superconducting DX separator dipoles in the IRs, which currently limit the maximum beam energy, will be removed to allow for an energy increase to $275 \mathrm{GeV}$.

Because eRHIC has to operate a over wide center-of-mass energy range both beam energies have to be varied in order to accomplish this. Since the proton beam is not ultra-relativistic, changing its energy results in a non-negligible velocity change. To keep the beams in the two rings synchronized, this velocity change has to be compensated by a corresponding variation of the circumference. In the energy range from 100 to $275 \mathrm{GeV}$ this is accomplished by adjusting the orbit radius within the magnet aperture. However, this method cannot be applied below $100 \mathrm{GeV}$ since the required radial change becomes much larger. Instead, an inner "BLUE" arc will be used in the sextant between IRs 2 and 12. The bending radius of an inner arc is about $90 \mathrm{~cm}$ smaller than that of an outer arc, resulting in a circumference reduction by approximately $90 \mathrm{~cm}$. This smaller circumference corresponds to a beam energy of $41 \mathrm{GeV}$.

Increasing the number of injected bunches three-fold, from the present 110 bunches to 330 in eRHIC, requires new, faster injection kickers. These kickers will be about $25 \mathrm{~m}$ long and will not fit into the present injection area in the arc near the IR6 straight section. Instead, the existing "BLUE" arc from IR6 to IR4 will be utilized to extend the existing transfer line and transport bunches to a new injection area in the IR4 straight section.

The increased number of short, intense bunches would lead to unacceptably high thermal load on the cryo system due to resistivity of the stainless steel beam pipes of the superconducting magnets. A thin copper coating will be applied in-situ to improve the conductivity of the pipe. In addition, a layer of amorphous carbon will be applied in order to reduce the secondary electron yield and therefore suppress electron clouds. 


\section{Beam dynamics}

Beam-beam effects in eRHIC have been studied in both weak-strong and strong-strong simulations $[11,12]$. Weak-strong simulations for the electron beam were used to determine the optimum working point around (.08/.06). Slow emittance growth in the hadron beam was examined using long-term weak-strong tracking. No evidence of such growth was found in head-on simulations; the effect of crab crossing is still under investigation.

With the parameters listed in Table 3 no signs of coherent beam-beam effects were observed in strong-strong simulations. When the bunch intensities were gradually increased the threshold for the onset of the instability was determined to be at double the design intensity in both beams. Coherent beam-beam effects are therefore expected to be not an issue in eRHIC.

The dynamic aperture in the electron storage ring has been assessed in the $10 \mathrm{GeV}$ configuration, i.e. with a FODO cell phase advance of 60 degrees. After optimization of the sextupole families in the arcs a dynamic aperture of at least $10 \sigma$ in all three dimensions was achieved. The off-momentum dynamic aperture is limited at $12 \sigma$ due to the second order chromaticity, which drives particles with large momentum offset into the integer resonance. The on-momentum dynamic aperture was found to be limited to approximately $20 \sigma$ by amplitude dependent tune shift. These results were obtained in an ideal machine without magnet imperfections or beam-beam effects. On the other hand, non-chromatic sextupoles in the straight ssections have not yet been explored either.

Long term tracking has been performed in the hadron ring lattice to determine the dynamic aperture, including beam-beam effects and multipole errors. With systematic multipole errors in the IR magnets limited to $2 \times 10^{-4}$ units at a reference radius of $25 \mathrm{~mm}$, the dynamic aperture measured in RMS beam sizes - practically equals the one found in tracking studies for the present RHIC lattice. Since this dynamic aperture is sufficient for successful RHIC operation, we conclude that it will also be sufficient for eRHIC.

\section{Polarization}

eRHIC needs to store highly polarized proton and electron beams with arbitrary spin patterns. RHIC has already achieved proton polarization levels over 60 percent at $255 \mathrm{GeV}$. For eRHIC, four more Siberian snakes will be added to the "YELLOW" ring, for a total of six. This is expected to improve polarization preservation on the ramp, resulting in about 70 percent polarization at $275 \mathrm{GeV}$.

The electron ring needs to store bunches with spin parallel and anti-parallel to the main guiding field simultaneously. Due to the Sokolov-Ternov effect bunches with their spins parallel to the dipole field will slowly depolarize and eventually build up polarization anti-parallel to the main guiding field. To keep high polarization levels in both orientations it is therefore necessary to replace entire bunches at a rate much faster than the Sokolov-Ternov time constant. With the shortest Sokolov-Ternov time being about 26 minutes, replacing each individual bunch after about 5 minutes yields sufficiently high polarization, assuming an initial polarization of 85 percent.

In addition to the Sokolov-Ternov effect, polarization is also affected by spin diffusion. In order to maintain an average polarization of 63 percent, spin diffusion effects have to be minimized 
such that the equilibrium polarization - for spins anti-parallel to the dipole field - reaches 50 percent. Spin tracking studies [13] show that this can be achieved by careful spin matching [15] between the spin rotators in the interaction region. Further improvements are expected by longitudinal spin matching and the application of harmonic bumps. However, the effect of the beam-beam interaction on electron polarization still needs to be investigated.

\section{IR layout}

The eRHIC interaction region design fulfills a number of challenging requirements:

- Small $\beta^{*}$ for high luminosity;

- Limited contribution of the IR magnets to the machine chromaticity;

- Large low- $\beta$ quadrupole apertures to accommodate forward-scattered protons and outgoing synchrotron radiation;

- Maximized detector acceptance, limiting the beam divergence;

- No accelerator magnets within $\pm 4.5 \mathrm{~m}$ of the interaction point;

- 22 mrad crossing angle for fast, dipole-free beam separation, compensated by crab crossing;

- Minimum synchrotron radiation background, requiring a long, dipole-free section upstream of the detector, a synchrotron radiation absorber far from the IP on the downstream side, and a dedicated system of synchrotron radiation masks;

- Accomodation of spin rotators, and proper spin matching;

- Space for a luminosity monitor, a neutron detector, and "Roman Pots".

Figure 4 shows the eRHIC interaction region layout with its multi-stage separation scheme - electrons from protons, protons from forward neutrons, and electrons from Bethe-Heitler photons used for the luminosity monitor. This very constrained system requires novel magnet types in the IR.

\section{Initial operating parameters}

The eRHIC design parameters are quite ambitious. Experience at other facilities has shown that reaching ambitious design parameters may take several years of operations. We have therefore developed a parameter set that can be reached with very little commissioning time, while it still fulfills the minimum requirements for an EIC, namely a luminosity of $10^{33} \mathrm{~cm}^{-2} \mathrm{sec}^{-1}$ and a centerof-mass energy of $105 \mathrm{GeV}$. Since this parameter set calls for a maximum electron beam energy of only $10 \mathrm{GeV}$ and a reduced electron beam current, $3 \mathrm{MW}$ of RF power is sufficient to reach these parameters, which could be realized by installing a reduced number of RF cavities in the electron storage ring, thus reducing the initial cost of the machine. Full luminosity performance would then be reached by gradually adding RF cavities, and ultimately strong hadron cooling. 


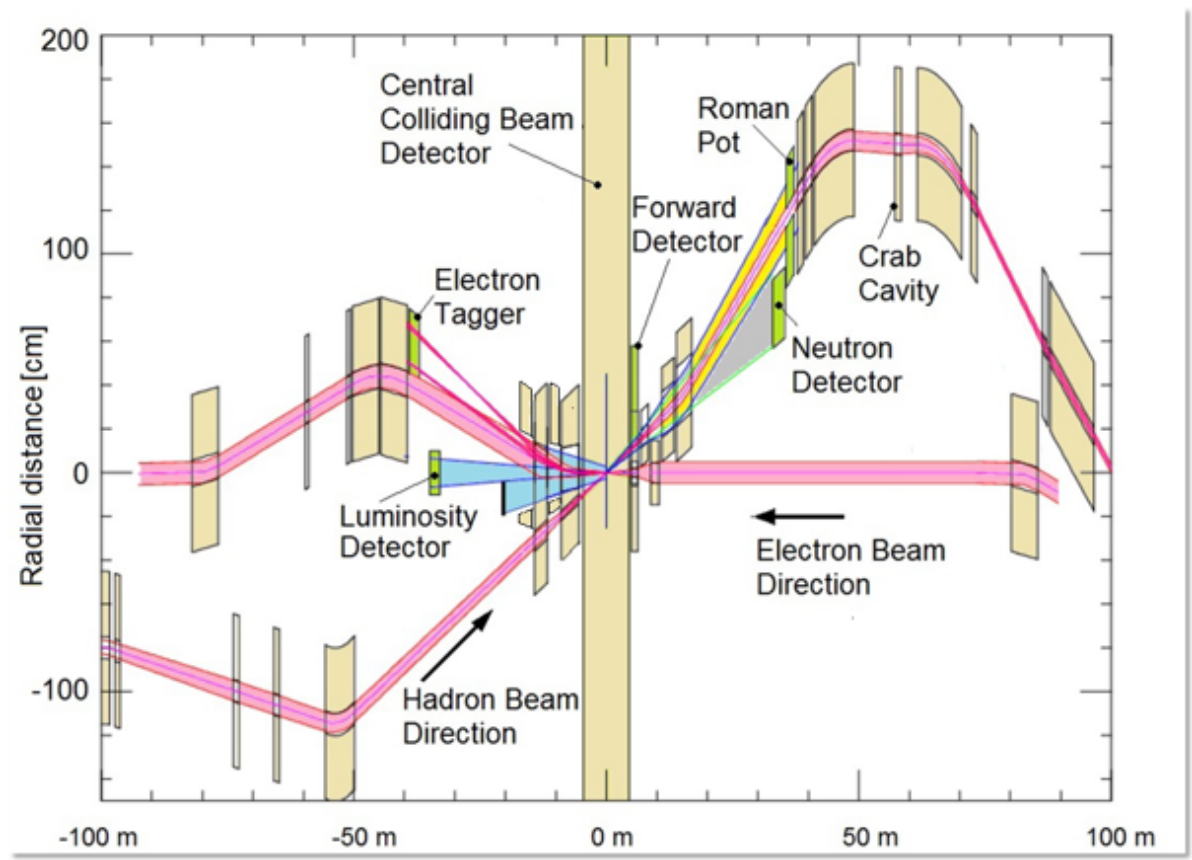

Figure 4: eRHIC interaction region layout

\section{Summary}

The proposed eRHIC design reaches a peak luminosity of $1.05 \times 10^{34} \mathrm{~cm}^{-2} \mathrm{sec}^{-1}$, and has a broad center-of-mass energy range from 29 to $141 \mathrm{GeV}$. However, reaching this high luminosity requires strong hadron cooling to counteract emittance growth due to intrabeam scattering with a time constant of about $2 \mathrm{~h}$. Since strong hadron cooling at high energies is a considerable risk, an alternate set of design parameters has been developed to mitigate this risk. This parameter set, which is based around an IBS growth time of about 9 hours - similar to present RHIC - reaches a peak luminosity of $4.4 \times 10^{33} \mathrm{~cm}^{-2} \mathrm{sec}^{-1}$ without the need for cooling.

\section{References}

[1] A. Accardi et al, Electron Ion Collider: The Next QCD Frontier, Eur. Phys. J., A52(9):268, 2016. arXiv:1212.101, doi:10.1140/epja/i2016-16268-9

[2] KEKB B-Factory Design Report, KEK Report 95-7

[3] HERA - A Proposal for a Large Electron Proton Colliding Beam Facility at DESY DESY-HERA-81-10, 1981

[4] V. Ranjbar et el., Spin resonance free electron ring injector, Phys. Rev. Accel. Beams 21, 111003, 2018

[5] CESR operating parameters, https://www/classe.cornell.edu/Research/CESR/OperatingParameters.html

[6] K. Oide, KEKB B-factory, the luminosity frontier, Prog. Theor. Phys., 122:69-80, 2009 
Table 5: Beam parameters during initial operation.

\begin{tabular}{lcc}
\hline \hline parameter & proton & electron \\
\hline center-of-mass energy [GeV] & \multicolumn{2}{c}{105} \\
energy [GeV] & 275 & 10 \\
number of bunches & \multicolumn{2}{c}{290} \\
particles per bunch [10 ${ }^{10}$ ] & 10.2 & 22.0 \\
beam current [A] & 0.37 & 0.8 \\
horizontal emittance [nm] & 17.9 & 20.0 \\
vertical emittance [nm] & 8.5 & 4.2 \\
hor. $\beta$-function at IP [cm] & 90 & 81 \\
vert. $\beta$-function at IP [cm] & 5.9 & 12.1 \\
hor./vert. fractional tunes & $0.3 / 0.31$ & $0.09 / 0.12$ \\
RMS hor. divergence at IP [mrad] & 0.141 & 0.157 \\
RMS vert. divergence at IP [mrad] & 0.380 & 0.186 \\
hor. beam-beam parameter & 0.0079 & 0.1 \\
vert. beam-beam parameter & 0.0029 & 0.085 \\
IBS growth time long./hor. [h] & $8 / 18$ & N/A \\
synchrotron radiation power [MW] & N/A & 2.95 \\
RMS bunch length [cm] & 9.9 & 1.4 \\
hourglass and crab reduction factor & \multicolumn{2}{c}{0.74} \\
luminosity [10 ${ }^{33}$ cm $^{-2}$ sec ${ }^{-1}$ ] & \multicolumn{2}{c}{1.05} \\
\hline \hline
\end{tabular}

[7] E.D. Bloom et al., The PEP-II asymmetric B factory: Design details and R\&D results, Conf. Proc., C940627:464-466, 1994

[8] J. Seeman et al., Performance of the PEP-II B-Factory Collider at SLAC, Conf. Proc., C0505161:2369, 2005

[9] V. N. Litvinenko and Y. S. Derbenev, Coherent Electron Cooling, Phys. Rev. Lett., 102:114801, 2009. doi:10.1103/PhysRevLett.02.114801

[10] D. Ratner, Microbunched Electron Cooling for High-Energy Hadron Beams, Phys. Rev. Lett., 111(8):084802, 2013. doi:10:1103/PhysRevLett.111.084802

[11] Y. Luo et al., "Study of Crabbed Collision in the eRHIC Design with a combination of Strong-Strong and Weak-Strong Beam-Beam Simulations”, Proc. IPAC'18, Vancouver, BC

[12] Y. Hao et al., "Study Progress of the Coupling Resonance of the Crab Crossing Scheme in Electron-Ion Collider", Proc. IPAC'18, Vancouver, BC

[13] E. Gianfelice-Wendt, Polarization Studies for the eRHIC Electron Storage Ring, these Proc.

[14] F. Meot et al., "Polarization at eRHIC EIC Storage Rings, Numerical Studies”, Proc. IPAC'18, Vancouver, BC

[15] V. Ptitsyn, Spin matching of interaction region with solenoidal spin rotators, these Proc. 